\title{
Dimensional dominance and adult shift learning
}

\author{
JULIUS 0. C. OZIOKO and RICHARD B. MAY \\ University of Victoria, Victoria, British Columbia, Canada
}

\begin{abstract}
Forty-eight adults learned intradimensional (ID) or extradimensional (ED) shifts in a total change paradigm under one of two conditions of dimensional dominance and one of two conditions of irrelevant dimension variability. Results showed a significant dominance main effect in initial training and significant Dominance by Shift and Variability by Shift interactions in shift training. When shifts were to a high-dominant dimension, there was no appreciable difference between ID and ED shifts. When shifts were to a low-dominant dimension, ID shift was easier than ED shift. The ID shift was learned faster than the ED shift when irrelevant dimensions in shift varied within trials. No reliable difference was found between ID and ED shifts when irrelevant dimensions varied between trials. These results support Esposito's (1975b) conclusion that the same principles guide shift behavior in children and adults.
\end{abstract}

Esposito (1975a, b) suggested that for adults the apparent ease of intradimensional (ID) shifts over extradimensional (ED) shifts is partially a function of dimensional dominance. Several studies have shown that children find shifts to a high-dominant dimension easier than shifts to a low-dominant dimension (e.g., May \& Fernandez, 1974). Esposito's work relating dominance to adult shift learning indicated that adults show the same type of discrimination shift behavior which is thought to characterize children's shift learning.

In addition, Esposito (1975a) suggested that shift performance may be a function of irrelevant dimension variability. While this variable has received little attention, Dickerson, Wagner, and Campione (1970) found that 5-year-olds learned ID shifts faster than ED when the irrelevant dimensions varied within trials and that the ID-ED difference was negligible when the irrelevant dimensions varied between trials (constant within trials). Shepp and Gray (1971) and Zeaman and Denegre (1967) reported somewhat similar findings with kindergarten children and retardates, respectively. However, this variable has not been explicitly dealt with using adults as subjects.

It has been suggested (e.g., Kendler \& Kendler, 1962) that age differences in shift learning may be indicative of differences in basic learning processes between young children compared with adults. However, the similarity of results reported by Esposito (1975b) with adults and Dickerson et al. (1970) with children suggest that dimensional dominance has a very similar effect upon the shift learning of both children and adults. One purpose of the present study was to replicate Esposito's findings with adults. A second purpose was to assess the generality of

This research was supported by a junior fellowship to the first author from the University of Nigeria. The research was submitted as part of a MA thesis carried out by the first author and supervised by the second author. J. O. C. Ozioko is now at the University of Nigeria, Nsukka, Nigeria. Reprints may be obtained from R. B. May, University of Victoria, Victoria, British Columbia, Canada. the effects of irrelevant dimension variability as studied by Dickerson et al. (1970). According to Esposito (1975a), adults should show the same ID-ED differences as children and we should therefore be able to replicate the results of Dickerson et al. with adults.

\section{METHOD}

\section{Subjects and Stimuli}

The subjects were 48 volunteer undergraduates attending the University of Victoria. There were 24 men and 24 women whose ages ranged from 19 to 25 years, with a mean of 22 years.

The stimuli consisted of two decks of white $3 \times 5$ in. $(7.6 \times$ $12.7 \mathrm{~cm}$ ) cards. Each card contained line drawings of geometric figures copied from those used by Esposito (1973). Esposito's 16-card decks were expanded to 32 cards each to completely counterbalance stimulus values on each of five dimensions. Dimensions and values in the training (preshift) deck were form (hexagon or triangle), number of forms (three or four), number of dots within each form (one or two), size of the forms $[1 / 2$ in. or $1 / 4$ in. $(1.3$ or $.6 \mathrm{~cm})$ diam] , and position of the forms (vertical center or top and bottom edge). The dimensions and values used in the shift deck were form (circle or square), number of forms (one or two), number of dots within a form (three or four), size of forms [1 $1 \frac{1 / 4}{\mathrm{in}}$ or $3 / 4$ in. $(3.2$ or $1.9 \mathrm{~cm})$ diam], and position of the forms on a card (horizontal center or vertical sides). Each stimulus card in training and shift displayed one value from each dimension, for a total of five values per card, and decks were constructed so that all dimensions were orthogonal.

\section{Procedure}

Preliminary scaling of the training deck was carried out with 20 undergraduates who did not take part in the shift study. Each of these subjects sorted the deck four times and a record was kept of the order in which dimensions were selected as a basis for sorting. These data were used to identify the form dimension (mean rank $=1.5$ ) and the position dimension (mean rank $=4.1$ ) as having highest and lowest dominance, respectively. The stimulus cards were then taped together in pairs to facilitate shuffling of pairs to randomize order of presentation for each subject and for successive presentations of a given deck. A trial was defined as presentation of a single pair of cards followed by a choice response to one member of the pair as "correct."

Subjects were tested individually while sitting at a table opposite the experimenter. They were told that they would be shown 16 pairs of cards and that each card contained a geometric figure which could vary in several ways. One property of each 
figure was relevant, or correct, and the other properties were irrelevant. The task was to pick the card which contained the correct stimulus property, with verbal correct-incorrect feedback from the experimenter after each choice. In training, stimulus pairs were presented one at a time until 14 consecutive correct responses were made. Subjects were given the shift task with no break in continuity of the trials.

Following the total change paradigm (cf. Esposito, 1975a), the shift task involved the same five dimensions used in initial training but employed all new values (cues) for each dimension. In ID shift the dimension which had been relevant during preshift remained relevant, while under ED shift, subjects who had received form as relevant had position relevant. Similarly, subjects who had received position relevant in preshift subsequently had form relevant in shift. Under the conditions of within-trial variation, all five dimensions varied within each trial. Under between-trial variation, two dimensions (form and position) were varied within each trial, while the other three dimensions varied only between trials. Shift training was continued until 14 consecutive correct responses were made.

For the shift task, form-relevant and position-relevant groups were randomly divided to receive either ID or ED shifts; half of each of these groups had the relevant dimension (form or position) and one irrelevant dimension (form or position) vary within trials, with the other three dimensions varying between trials. Thus, the design was a 2 (dimensional diminance) by 2 (type of shift) by 2 (locus of irrelevant variability) factorial, with six subjects randomly assigned to each cell (with the restriction that an equal number of men and women were in each group).

\section{RESULTS}

Table 1 gives the means and standard deviations on errors to criterion in both initial training and shift. These data were submitted to 2 dominance (form/position) by 2 shifts (ID/ED) by 2 locus of variability (within/ between) analysis of variance. Preliminary examination of the data revealed no reliable effects associated with sex of subject either in initial training or in shift.

\section{Initial Training}

Analysis of error scores in preshift revealed no significant main effects for type of shift or locus of variability and no interactions between any variables ( $\mathrm{F}<1$ in each case). However, there was a substantial effect due to dimensional dominance $[\mathrm{F}(1,40)=168.47$, $\mathrm{p}<.001]$ which accounted for $80 \%$ of the total variance. Consistent with similar studies using children as subjects (Wolff, 1967), the form-relevant group (mean = 1.88) made fewer errors than the position-relevant group $($ mean $=7.00)$.

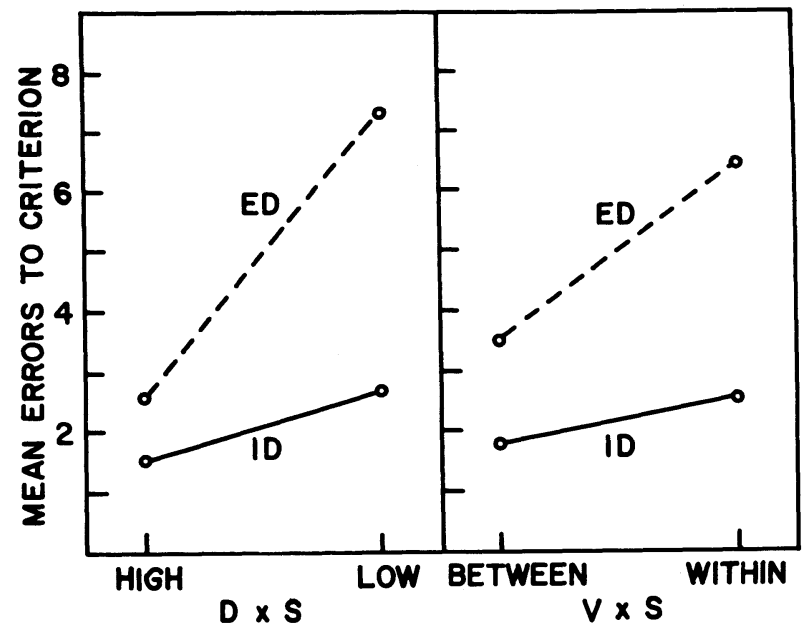

Figure 1. Plot of the Dominance by Shift $(D \times S)$ and Variability by Shift $(V \times S)$ interactions.

\section{Shift}

Analysis of variance of error scores on the shift task revealed three significant main effects (shift, $p<.001$; dominance, $\mathrm{p}<.001$; variability, $\mathrm{p}<.001)$. In addition, the Dominance by Shift interaction $[F(1,40)=23.52$, $\mathrm{p}<.001]$ and the Variability by Shift interaction $[F(1,40)=7.95, p<.05]$ were both significant. A plot of these two interactions is given in Figure 1. All other effects were negligible $(p>.10)$.

As shown in Figure 1, when all four irrelevant stimulus dimensions varied within trials, the ED shift was learned slower than the ID shift $(p<.01)$. When three of the dimensions varied only between trials, there was no appreciable difference between types of shift $(p>.05)$. In the Dominance by Shift interaction, when the relevant dimension was low dominant, ID shift was learned faster than ED shift $(p<.001)$. However, when the relevant dimension was high dominant, there was little difference between ID- and ED-shift performance $(\mathrm{p}>.10)$.

\section{DISCUSSION}

The present results show that adults learn ID shifts faster than ED shifts, but only under certain circumstances, that is, when the shift task has a low-dominant dimension relevant or when the irrelevant dimension varies within trials. These ID-ED

Table 1

Means and Standard Deviations of the Error Scores on the Preshift and Postshift Tasks

\begin{tabular}{|c|c|c|c|c|c|c|c|c|}
\hline \multirow[b]{3}{*}{ Tasks } & \multicolumn{4}{|c|}{$\begin{array}{l}\text { Variable-Between Trial } \\
\text { Dominance }\end{array}$} & \multicolumn{4}{|c|}{$\begin{array}{l}\text { Variable-Within Trial } \\
\text { Dominance }\end{array}$} \\
\hline & \multicolumn{2}{|c|}{ High } & \multicolumn{2}{|c|}{ Low } & \multicolumn{2}{|c|}{ High } & \multicolumn{2}{|c|}{ Low } \\
\hline & ID* & ED & ID & ED & ID & ED & ID & ED \\
\hline $\begin{array}{l}\text { Preshift } \\
\text { SD }\end{array}$ & $\begin{array}{r}1.83 \\
.98\end{array}$ & $\begin{array}{r}1.50 \\
.55\end{array}$ & $\begin{array}{l}7.00 \\
1.41\end{array}$ & $\begin{array}{l}7.17 \\
1.72\end{array}$ & $\begin{array}{l}2.00 \\
1.27\end{array}$ & $\begin{array}{l}2.17 \\
1.17\end{array}$ & $\begin{array}{l}6.83 \\
1.17\end{array}$ & $\begin{array}{l}7.00 \\
2.10\end{array}$ \\
\hline $\begin{array}{l}\text { Postshift } \\
\text { SD }\end{array}$ & $\begin{array}{r}1.67 \\
.75\end{array}$ & $\begin{array}{l}1.83 \\
1.17\end{array}$ & $\begin{array}{l}2.33 \\
1.75\end{array}$ & $\begin{array}{l}5.50 \\
1.05\end{array}$ & $\begin{array}{r}2.00 \\
.89\end{array}$ & $\begin{array}{l}3.67 \\
1.87\end{array}$ & $\begin{array}{l}2.83 \\
1.47\end{array}$ & $\begin{array}{l}9.17 \\
1.47\end{array}$ \\
\hline
\end{tabular}

*ID = intradimensional shift $; E D=$ extradimensional shift. 
shift differences are consistent with attention theories (e.g., Fisher \& Zeaman, 1973; Zeaman \& House, 1963). Since the response requirements of the two shifts seem to be different, the ED-shift task may be difficult because subjects are required to attend to a dimension that has previously been ignored due to low perceptual dominance and due to lack of reinforcement.

The importance of dimensional dominance in adult discrimination learning was confirmed. The ED shift was more difficult than the ID shift only when the shift was to a low-dominant relevant dimension. This outcome is consistent with findings reported by Esposito (1975b) with adults and Dickerson et al. (1970) with children as subjects. The performance of subjects trained on a high-dominant dimension (form) and maintained on that same dimension during the shift (ID group) did not differ reliably from the performance of subjects trained on a lowdominant (position) and shifted to a high-dominant dimension (ED group). This finding and the significant influence of dimensional dominance in initial training are consistent with Wolff's (1967) suggestion that almost all subjects, regardless of age, dimensionalize stimuli in discrimination tasks. Seitz and Weir (1971) have proposed that older children and adults should be mixed dimensional responders and not have a dominant dimension. However, the present data support the notion that dimensional dominance is stable not only in children but also in adults and that this variable has a similar influence upon discrimination shift behavior of both children and adults.

Our results extend the effects of within-trial vs betweentrial variation in irrelevant dimensions from children (Dickerson et al., 1970; Shepp \& Grey, 1971) to adults, as recommended by Esposito (1975a). The differences between ID and ED shifts are negligible when irrelevant dimensions vary between trials, but ED shifts are much harder when irrelevant dimensions vary within trials.

The negligible difference between types of shift under between-trial variation may be due to the noncompetitiveness or within-trial constancy of the irrelevant dimensions. The relevant dimension appears to suffer no effective competition from irrelevant dimensions in this condition and appears to control performance to about the same extent in both ID- and EDshift conditions. This is not inconsistent with Johnson's (1966) report that an increase in the number of irrelevant cues will retard ED-shift performance but have little effect on ID-shift learning.

\section{REFERENCES}

Dickerson, D. J., WAgner, J. F., \& Campione, J. Discrimination shift performance of kindergarten children as a function of variation of the irrelevant dimension. Developmental Psychology, 1970, 3, 229-235.

Esposito, N. J. Effects of stimulus novelty and dimensional saliency in human shift learning. Journal of Experimental Psychology, 1973, 98, 264-270.

Esposito, N. J. Review of discrimination shift learning in young children. Psychological Bulletin, 1975, 82, 432-455. (a).

Esposito, N. J. Effect of dimensional preference on adult shift learning: Some developmental implications. Developmental Psychology, 1975, 11, 851-852. (b)

Fisher, M. A., \& Zeaman, D. An attention-retention theory of retardate discrimination learning. In N. R. Ellis (Ed.), International review of research in mental retardation (Vol. 6). New York: Academic Press, 1973.

Johnson, P. J. Factors affecting transfer in concept identification problems. Journal of Experimental Psychology, 1966, 72, 655-660.

KendleR, H. H., \& Kendler, T. S. Vertical and horizontal processes in problem solving. Psychological Review, 1962, 69, 1-16.

MAY, R. B., \& Fernandez, D. Dimensional dominance and extradimensional shifts. Perceptual and Motor Skills, 1974, 38, 979-985.

SEITz, V., \& WEIR, M. W. Strength of dimensional preferences as a prediction of nursery school children's performance on a concept-shift task. Journal of Experimental Child Psychology, $1971,12,370-386$.

Shepp, B. E., \& Gray, V. A. Some effects of variable-within and variable-between irrelevant stimuli on dimensional learning and transfer. Journal of Experimental Psychology, $1971,89,32-39$

Wolff, J. L. Concept-shift and discrimination-reversal learning in humans. Psychological Bulletin, 1967, 68, 369-408.

Zeaman, D., \& Denegre, J. Variability of irrelevant discriminative stimuli. Journal of Experimental Psychology, 1967, 73, 574-580.

ZEAMAN, D., \& HousE, B. J. The role of attention in retardate discrimination learning. In N. R. Ellis (Ed.), Handbook of mental deficiency. New York: McGraw-Hill, 1963.

(Received for publication December 9, 1976.) 